\title{
Modelling of Errors and Uncertainties in Photoacoustic Tomography using a Bayesian Framework
}

\author{
Tanja Tarvainen $^{1,2}$, Teemu Sahlström ${ }^{1}$, Jenni Tick ${ }^{1}$, Aki Pulkkinen ${ }^{1}$ \\ ${ }^{1}$ Department of Applied Physics, University of Eastern Finland, P.O. Box 1627, 70211 Kuopio, Finland \\ ${ }^{2}$ Department of Computer Science, University College London, Gower Street, London WC1E 6BT, United \\ Kingdom \\ tanja.tarvainen@uef.fi
}

\begin{abstract}
Photoacoustic tomography is studied in the framework of Bayesian inverse problems. Modelling of errors and uncertainties using Bayesian approximation error modelling is investigated. The approach is tested with simulations. (c) 2019 The Author(s) OCIS codes: $100.3190,170.3010,170.5120$
\end{abstract}

\section{Introduction}

Photoacoustic tomography (PAT) [1,2] is an imaging modality combining optical contrast with high spatial resolution of ultrasound. In PAT, imaged tissue is illuminated by a short pulse of visible or near-infrared light. The absorption of light generates local increases in pressure that propagates through the tissue as an acoustic wave and can be measured on the boundary of the target by ultrasound sensors. In the inverse problem of PAT, the initial pressure distribution is estimated from the measured ultrasound time-series. In this work, the PAT inverse problem is approached in the framework of Bayesian inverse problems [3,4]. We study modelling of errors and uncertainties in PAT measurement situation using Bayesian approximation error modelling [3].

\section{Methods}

Let us denote the discretised initial pressure distribution by $p_{0} \in \mathbb{R}^{n}$ and let us denote the measured photoacoustic time-series by $p_{t} \in \mathbb{R}^{m}$. In PAT, the observation model with an additive noise model is of the form

$$
p_{t}=K_{\delta} p_{0}+e
$$

where $K_{\delta} \in \mathbb{R}^{m \times n}$ is a forward model which maps the initial pressure to the measurable data and $e \in \mathbb{R}^{m}$ denotes the noise. In this paper, photoacoustic wave propagation is modelled using a linear, homogeneous wave equation. The solution of the wave equation is numerically approximated using $k$-space time-domain method implemented with the k-Wave MATLAB toolbox [5].

In practice, the measurements or the forward model may contain errors or uncertainties, for example, due to uncertainties in sensor positions or object shape or due to using approximations in the forward modelling. In that case, the observation model can be written in the from

$$
p_{t}=K p_{0}+\varepsilon+e
$$

where $\varepsilon=K_{\delta} p_{0}-K p_{0}$ is the modelling error which describes the discrepancy between the exact forward model $K_{\delta}$ and an approximate or inexact forward model $K \in \mathbb{R}^{m \times n}$ [3].

In the inverse problem of PAT, the initial pressure distribution $p_{0}$ is estimated from the measured photoacoustic wave $p_{t}$. Let us approximate the noise statistics and the prior for the initial pressure as mutually independent and Gaussian distributed, $e \sim \mathscr{N}\left(\eta_{e}, \Gamma_{e}\right), p_{0} \sim \mathscr{N}\left(\eta_{p_{0}}, \Gamma_{p_{0}}\right)$. Further, let us approximate the modelling error and total error $n=\varepsilon+e$ as Gaussian, $\varepsilon \sim \mathscr{N}\left(\eta_{\varepsilon}, \Gamma_{\varepsilon}\right), n \sim \mathscr{N}\left(\eta_{n}, \Gamma_{n}\right)$ and ignore the mutual dependence of the modelling error and initial pressure. Now, in the case of a linear observation model and Gaussian distributed parameters, the posterior density is also a Gaussian distribution

$$
p_{0} \mid p_{t} \sim \mathscr{N}\left(\eta_{p_{0} \mid p_{t}}, \Gamma_{p_{0} \mid p_{t}}\right)
$$

where

$$
\begin{aligned}
\eta_{p_{0} \mid p_{t}} & =\left(K^{\mathrm{T}} \Gamma_{n}^{-1} K+\Gamma_{p_{0}}^{-1}\right)^{-1}\left(K^{\mathrm{T}} \Gamma_{n}^{-1}\left(p_{t}-\eta_{n}\right)+\Gamma_{p_{0}}^{-1} \eta_{p_{0}}\right) \\
\Gamma_{p_{0} \mid p_{t}} & =\left(K^{\mathrm{T}} \Gamma_{n}^{-1} K+\Gamma_{p_{0}}^{-1}\right)^{-1}
\end{aligned}
$$

where $\eta_{p_{0} \mid p_{t}}$ and $\Gamma_{p_{0} \mid p_{t}}$ are the mean and covariance of the posterior distribution [4]. 

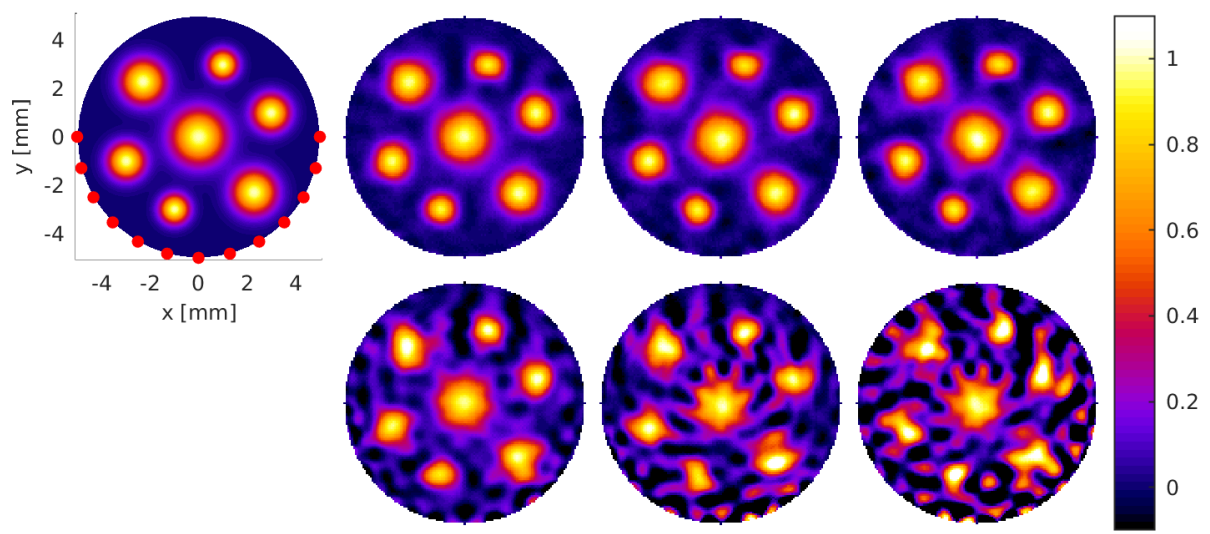

Fig. 1. True (simulated) initial pressure (first column) and the reconstructed initial pressure distributions from data simulated in situations in which the sensor locations were perturbed $\pm(0.5-1)$ degrees (second column), $\pm(1-2)$ degrees (third column) and $\pm(1.5-3)$ degrees (fourth column). The top row show results when the uncertainties have been modelled and the bottom row show the results when the uncertainties have not been modelled. The red dots in the left image indicate the unperturbed sensor locations that were used in the solution of the inverse problem.

\section{Results}

We studied Bayesian approximation error modelling in compensating uncertainties in PAT measurement situation. An example of the estimated mean of the posterior distributions in a situation where the locations of the ultrasound sensors were mismodelled is shown in Fig. 1. In simulations, a two-dimensional circular domain with a diameter of $10 \mathrm{~mm}$ was used. 19 ideal point-like sensors were equally distributed on the bottom half of the region with an angular separation of 10 degrees. Data was simulated using sensor positions which were varied to create uncertainties. In the inverse problem, the unperturbed sensor locations were used. The inverse problem was solved with the enhanced error model (2) by solving the mean and covariance of the posterior distribution by (4)-(5). Statistics of the modelling error $\varepsilon$ was constructed by computing approximations for the mean and covariance from samples of the modelling error. These samples were generated by solving the forward model using samples drawn from a distribution of sensor locations and prior for initial pressure. For comparison, the inverse problem was solved assuming $\varepsilon=0$, i.e. not taking into account the uncertainties in the sensor locations.

\section{Conclusions}

The results show that the Bayesian approximation modelling can be used to compensate for modelling errors such as mismodelled sensor locations in a PAT measurement setup.

\section{Acknowledgements}

This work has been supported by the Academy of Finland (projects 286247, 314411 and 312342 Centre of Excellence in Inverse Modelling and Imaging) and Jane and Aatos Erkko Foundation.

\section{References}

1. P. Beard, "Biomedical photoacoustic imaging," Interface Focus 1, 602-631 (2011).

2. L. V. Wang and J. Yao "A practical guide to photoacoustic tomography in the life sciences," Nat Methods 13, 627-638 (2016).

3. J. Kaipio and E. Somersalo, Statistical and Computational Inverse Problems. (Springer Science \& Business Media, 2006).

4. J. Tick, A. Pulkkinen, and T. Tarvainen, "Image reconstruction with uncertainty quantification in photoacoustic tomography," J Acoust Soc Am 139, 1951-1961 (2016).

5. B. E. Treeby and B. T. Cox, "k-wave: Matlab toolbox for the simulation and reconstruction of photoacoustic wave fields," J Biomed Opt 15, 021314 (2010). 